\title{
Secuencia de abordaje diagnóstico-terapéutico para el paciente intoxicado. Parte 2: tratamiento
}

\author{
Jorge G. Pérez-Tuñón, Mayré I. Bautista-Albiter*, Herminio Terán-Flores, Juan C. Pérez-Hernández, \\ José Padilla-Ochoa y Mireille D. Arango-Mathieu \\ Centro Toxicológico, Hospital Ángeles Lomas, Huixquilucan, Estado de México, México
}

\section{Resumen}

La presente publicación corresponde a la segunda parte de la revisión de la secuencia de abordaje diagnóstico-terapéutico para el paciente potencialmente intoxicado que hemos propuesto en una anterior publicación, misma en la que fueron abordados los pasos correspondientes a la estabilización del paciente, así como a la secuencia diagnóstica a partir del interrogatorio, la exploración física y los métodos de complemento diagnóstico. Por su parte, en la presente sección se desarrollan los pasos que corresponden al abordaje terapéutico, los cuales se subdividen en descontaminación, eliminación y antídoto.

Palabras clave: Intoxicación. Sobredosis. Antídotos.

\section{Sequence of diagnostic-therapeutic approach for the potentially intoxicated patient.} Part 2: Treatment

\begin{abstract}
Present publication corresponds to the second part of the review of the sequence of diagnostic-therapeutic approach for the potentially intoxicated patient, that we proposed in a previously publication, same in which the steps corresponding to the stabilization of the patient were approached, as well as the diagnostic sequence from the interrogation, the physical examination and the diagnostic complement methods. In this section develops the steps that correspond to the therapeutic approach, which are subdivided into decontamination, elimination and antidote.
\end{abstract}

Key words: Poisoning. Overdose. Antidotes.

\section{Introducción}

El abordaje de un paciente expuesto a una sustancia potencialmente tóxica involucra muchos aspectos que resultan vitales, por lo que hemos desarrollado una secuencia de abordaje diagnóstico-terapéutico para el paciente potencialmente intoxicado, la cual permite de forma didáctica contemplar todos los puntos indispensables en lo concerniente a estos pacientes (Tabla 1), haciendo hincapié en que el orden planteado busca priorizar los aspectos clave en el abordaje y no indicar una secuencia cronológica obligada que pudiera retrasar las maniobras terapéuticas, de tal modo que en la

\section{Correspondencia:}

*Mayré I. Bautista-Albiter

E-mail: toxicología_angeles@yahoo.com.mx

\footnotetext{
Disponible en internet: 25-11-2021

Rev Educ Investig Emer. 2021;3(4):207-214
www.medicinadeemergencias.com

Rev Educ Investig Emer. 2021;3(4):207-214
www.medicinadeemergencias.com

Fecha de recepción: 18-03-2021 Fecha de aceptación: 01-06-2021 DOI: 10.24875/REIE.21000021 CV. Este es un artículo open access
} bajo la licencia CC BY-NC-ND (http://creativecommons.org/licenses/by-nc-nd/4.0/). 
Tabla 1. Secuencia de abordaje diagnóstico-terapéutico para el paciente potencialmente intoxicado*

\begin{tabular}{|l|l|}
\hline 0 & Estabilización \\
\hline 1 & Interrogatorio \\
\hline 2 & Exploración física \\
\hline 3 & Complemento diagnóstico (laboratorio, gabinete) \\
\hline 4 & Descontaminación \\
\hline 5 & Eliminación \\
\hline 6 & Antídoto \\
\hline
\end{tabular}

*El presente abordaje deberá ajustarse a las necesidades individuales de cada paciente (no todos requieren descontaminación o eliminación ni existen antídotos para todos los xenobióticos).

medida de lo posible puedan llevarse a cabo simultáneamente. Por otro lado, debido a que la presente revisión ha sido dividida en dos secciones, invitamos al lector a consultar el trabajo Secuencia de abordaje diagnóstico-terapéutico para el paciente potencialmente intoxicado. Parte 1, donde se analiza la estabilización (paso 0), así como los pasos que corresponden al diagnóstico: interrogatorio (paso 1), exploración física (paso 2) y complemento diagnóstico (paso 3).

\section{Descontaminación}

En todo paciente que ha estado expuesto a un xenobiótico potencialmente tóxico es importante limitar el daño removiendo dicha sustancia del sitio de contacto antes de que se lleve a cabo la completa absorción, lo anterior, teniendo siempre en cuenta la protección del personal de salud en los casos que así se requiera. La descontaminación puede llevarse a cabo en diferentes superficies de absorción, siendo las más comunes la mucosa digestiva, la piel y los ojos ${ }^{1,2}$.

\section{Descontaminación gastrointestinal}

Se lleva a cabo mediante procedimientos que no se hallan exentos de complicaciones, por lo que su empleo debe individualizarse a cada paciente considerando sus riesgos y beneficios. Ello se logra al considerar el tipo de xenobiótico implicado, la cantidad, el tiempo desde la ingesta, la posibilidad de ingesta de más de un xenobiótico, el estado clínico del paciente, su edad, peso, talla y comorbilidades. En la tabla 2 se resumen las principales técnicas de descontaminación gastrointestinal ${ }^{3-14}$.
Por otro lado, la endoscopia es un método que llega a emplearse para la descontaminación gástrica cuando el xenobiótico se encuentra encapsulado (p. ej., baterías de disco) o cuando ha formado concreciones que difícilmente continuarán su tránsito a través del píloro ${ }^{15}$. Finalmente, en ocasiones es necesario descontaminar mediante gastrotomía si el xenobiótico no es removible por las técnicas anteriores, como en el caso de la ingesta de paquetes de droga que han perdido su integridad y existe el riesgo de una absorción masiva de esta o en pacientes con intoxicación por tabletas de hierro que han quedado adheridas a la mucosa, entre otros ${ }^{3}$.

\section{Descontaminación cutánea}

Para llevar a cabo la descontaminación de la piel, el personal de salud debe encontrarse protegido dependiendo de las características del xenobiótico que descontaminar. Dependiendo de las características del xenobiótico que descontaminará el nivel de protección necesario para la mayoría de los compuestos absorbibles a través de la piel corresponde al nivel $C$ de la Agencia de Protección Ambiental de EE.UU. (EPA), el cual consiste en un traje impermeable de cuerpo completo, botas, guantes y máscara con filtro, aunque existen sustancias que por su elevada volatilidad pueden requerir un nivel B (con sistema autónomo de respiración) o superior ${ }^{16}$. Con el personal de salud protegido, la ropa del paciente debe retirarse y disponerse en bolsas para su manejo como residuo peligroso. Posteriormente se realiza el lavado con abundante agua y jabón, con especial atención en pliegues, uñas y espacios interdigitales. Por otro lado, la exposición dérmica a metales alcalinos (grupo 1: litio, sodio, potasio, rubidio, cesio y francio) requiere descontaminación por barrido (cepillado en seco) o con soluciones alcalinas, ya que el uso de agua puede causar una reacción exotérmica que provoque lesiones secundarias ${ }^{17,18}$.

\section{Descontaminación oftálmica}

Cuando un xenobiótico potencialmente tóxico entra en contacto con los ojos puede ocurrir una lesión local o con menor frecuencia su absorción sistémica, por lo que es importante la identificación del xenobiótico implicado, así como la medición del pH ocular y el inicio inmediato de la descontaminación. Para ello, en ocasiones es necesaria la administración previa de un anestésico local como la tetracaína, sin embargo, cuando ello no es posible debe procederse 
Tabla 2. Técnicas de descontaminación gastrointestinal*

\begin{tabular}{|c|c|c|c|}
\hline Consideraciones & Lavado gástrico & Carbón activado (CA) & Irrigación intestinal total \\
\hline ¿Cómo actúa? & $\begin{array}{l}\text { Extrae el xenobiótico de la cámara } \\
\text { gástrica }\end{array}$ & $\begin{array}{l}\text { Se adhiere al xenobiótico } \\
\text { impidiendo su absorción }\end{array}$ & $\begin{array}{l}\text { Arrastra el xenobiótico por el } \\
\text { tracto digestivo hasta ser } \\
\text { evacuado }\end{array}$ \\
\hline \multirow[t]{2}{*}{ Indicaciones } & \multicolumn{3}{|c|}{$\begin{array}{l}\text { El xenobiótico ingerido es potencialmente tóxico, la vía aérea está protegida (en caso de paciente con deterioro } \\
\text { del estado de alerta), el beneficio es mayor que el riesgo y no existe un tratamiento alternativo más seguro o } \\
\text { más efectivo }\end{array}$} \\
\hline & $\begin{array}{l}\text { Tiempo de ingesta menor a } 2 \text { horas }^{\dagger} \text {, } \\
\text { el xenobiótico no es absorbible por } \\
\text { CA o lo supera en una relación } \\
\text { mayor a 1:10 (en gramos) }\end{array}$ & $\begin{array}{l}\text { El xenobiótico se adsorbe por CA y } \\
\text { permanece en el tracto } \\
\text { gastrointestinal }\end{array}$ & $\begin{array}{l}\text { El xenobiótico tiene un periodo de } \\
\text { latencia para su liberación, no se } \\
\text { adhiere al CA o su cantidad y/o } \\
\text { volumen lo superan }\end{array}$ \\
\hline Contraindicaciones & $\begin{array}{l}\text { Xenobiótico único, con antídoto } \\
\text { eficaz o absorbible por CA, } \\
\text { cáusticos, hidrocarburos, riesgo de } \\
\text { sangrado o perforación }\end{array}$ & $\begin{array}{l}\text { Xenobiótico con antídoto eficaz, no } \\
\text { absorbible por CA o con alto riesgo } \\
\text { de inhalación (hidrocarburos), } \\
\text { cáusticos y riesgo de sangrado o } \\
\text { perforación }\end{array}$ & $\begin{array}{l}\text { Xenobiótico con antídoto eficaz o } \\
\text { absorbible por CA, cáusticos, íleo, } \\
\text { oclusión intestinal, cirugía } \\
\text { gastrointestinal reciente, riesgo } \\
\text { de sangrado o perforación }\end{array}$ \\
\hline Material & $\begin{array}{l}\text { Niños: sonda calibre } 22 \text { a } 28 \mathrm{fr} \text {; } \\
\text { adultos: sonda calibre } 36 \text { a } 40 \mathrm{fr} \text {, } \\
\text { agua bidestilada }\end{array}$ & $\begin{array}{l}\text { CA, manitol u otro catártico, sonda } \\
\text { de calibre mediano o vaso para } \\
\text { administración }\end{array}$ & $\begin{array}{l}\text { Polietilenglicol } 3350 \text { o equivalente, } \\
\text { sonda de calibre mediano o vaso } \\
\text { para administración }\end{array}$ \\
\hline \multirow[t]{2}{*}{ Técnica } & \multicolumn{3}{|c|}{ 1. Verificar reflejos protectores de la vía aérea o intubar } \\
\hline & $\begin{array}{l}\text { 2. Paciente en decúbito lateral } \\
\text { izquierdo y Trendelemburg a } 15^{\circ} \\
\text { 3. La sonda se mide, se marca y se } \\
\text { introduce por la boca (no se fija) } \\
\text { 4. Alícuotas de } 10 \mathrm{ml} / \mathrm{kg} \text { de peso en } \\
\text { niños y de } 250 \mathrm{ml} \text { en adultos (se } \\
\text { ingresa y egresa por gravedad) } \\
\text { 5. Cuando el efluente es cristalino } \\
\text { se concluye el procedimiento }\end{array}$ & $\begin{array}{l}\text { 2. Elegir la vía de administración } \\
\text { (oral o por sonda) con base en } \\
\text { edad, estado neurológico y } \\
\text { cooperación del paciente } \\
\text { 3. Administrar a dosis de } 0.5 \text { a } \\
1 \mathrm{~g} / \mathrm{kg} \text { de peso en adultos y } \\
\text { niños, con una dilución de } 4 \mathrm{ml} \\
\text { de manitol al } 20 \% \text { (o equivalente) } \\
\text { por cada gramo de CA } \\
\text { 4. El carbón administrado no debe } \\
\text { retirarse }\end{array}$ & $\begin{array}{l}\text { 2. Elegir la vía de administración } \\
\text { (oral o por sonda) con base en } \\
\text { edad, estado neurológico y } \\
\text { cooperación del paciente } \\
\text { 3. Administrar a dosis de } 25 \text { a } \\
40 \mathrm{ml} / \mathrm{kg} \text { de peso/h en niños o } \\
\text { de } 1.5 \text { a } 2 \text { l/hora en adultos } \\
\text { 4. Cuando el efluente es cristalino } \\
\text { se concluye el procedimiento }\end{array}$ \\
\hline Complicaciones & $\begin{array}{l}\text { Vómitos, broncoaspiración, } \\
\text { aumento del vaciamiento gástrico } \\
\text { transpilórico (paso del xenobiótico } \\
\text { al duodeno), hipoxia, trauma } \\
\text { orofaríngeo, espasmo laríngeo y } \\
\text { desequilibrio hidroelectrolítico }\end{array}$ & $\begin{array}{l}\text { Náusea, vómitos, cólico, malestar } \\
\text { gastrointestinal, neumonitis por } \\
\text { aspiración, formación de bezoares } \\
\text { y oclusión intestinal }\end{array}$ & $\begin{array}{l}\text { Náusea, vómitos, } \\
\text { broncoaspiración, lesión de tracto } \\
\text { gastrointestinal, laringoespasmo, } \\
\text { distensión abdominal, cólico y } \\
\text { prurito anal }\end{array}$ \\
\hline
\end{tabular}

*Antes de iniciar cualquiera de estos procedimientos, el paciente debe contar con acceso venoso y con monitoreo hemodinámico.

${ }^{\dagger}$ Considerar xenobióticos que retrasen el vaciamiento gástrico como anticolinérgicos, opioides o aquellos que forman concreciones, en cuyo caso puede realizarse tardíamente el lavado gástrico.

Adaptada de Schwarz, 20173; Hoegberg, 2019'; Chyka, et al., 20055; Albertson, et al., 20116; Merigian, et al., 19907; Harchelroad, et al., 1989; ; Green, et al., 20049.

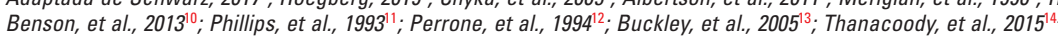

a realizar una irrigación continua, con abundante agua corriente o soluciones isotónicas en ambos ojos (conjuntivas, córnea, recesos y párpados) por al menos 15 a 20 minutos. Si hay lentes de contacto, estos deben ser retirados. El uso de blefarostato también es de utilidad si este no impide la adecuada descontaminación del párpado. Si se trató de un cáustico, al término se debe volver a medir el $\mathrm{pH}$ ocular y reiniciar el lavado de ser necesario. La consulta oftalmológica es requerida para la evaluación de daño estructural con lámpara de hendidura. Cabe señalar que debe evitarse realizar el lavado ocular con jeringa o a goteo continuo debido a que de esta manera no se logra la presión necesaria para el barrido del xenobiótico y, por el contrario, si se trata de un ácido o un álcali, es posible desencadenar una reacción exotérmica que incremente la lesión ${ }^{17-19}$.

\section{Eliminación}

Cuando no fue posible prevenir la absorción de un xenobiótico, debe considerarse su eliminación si dicho 
Tabla 3. Métodos para incrementar la eliminación de un xenobiótico

\begin{tabular}{|c|c|c|c|}
\hline Consideraciones & Alcalinización sérica-urinaria & $\begin{array}{l}\text { Diálisis gastrointestinal (dosis } \\
\text { múltiples de CA) }\end{array}$ & $\begin{array}{l}\text { Técnicas extracorpóreas (HD, } \\
\text { HF, HP) }\end{array}$ \\
\hline ¿Cómo actúa? & $\begin{array}{l}\text { Ioniza ácidos débiles impidiendo } \\
\text { su movilización entre las } \\
\text { membranas. De este modo se } \\
\text { bloquea la reabsorción tubular }\end{array}$ & $\begin{array}{l}\text { Impide circulación entero-hepática } \\
\text { (E-H), entero-entérica (E-E) y elimina } \\
\text { xenobióticos desde plexos } \\
\text { gastrointestinales }\end{array}$ & $\begin{array}{l}\text { Remueven xenobióticos a través } \\
\text { de una membrana siguiendo } \\
\text { gradientes osmóticos, } \\
\text { hidrostáticos o electroquímicos. }\end{array}$ \\
\hline \multirow[t]{2}{*}{ Indicaciones } & \multirow{2}{*}{$\begin{array}{l}\text { Xenobióticos con } \mathrm{pK}_{\mathrm{a}} \text { (constante } \\
\text { ácida de disociación): } \\
\text { salicilatos, fenobarbital, } \\
\text { metotrexato, clorpropamida, } \\
\text { ácido fórmico y herbicidas del } \\
\text { grupo clorofenoxi }\end{array}$} & \multicolumn{2}{|c|}{$\begin{array}{l}\text { Xenobióticos con bajo volumen de distribución }(<1 \mathrm{l} / \mathrm{kg}) \text {, cinética } \\
\text { monocompartimental y limitado aclaramiento renal }(<4 \mathrm{ml} / \mathrm{kg} / \mathrm{min})\end{array}$} \\
\hline & & $\begin{array}{l}\text { Con circulación E-H y/o E-E y } \\
\text { absorbibles por CA: } \\
\text { A: aspirina y salicilatos, antidepresivos } \\
\quad \text { cíclicos, amanitas, aminofilina y } \\
\quad \text { antipalúdicos } \\
\text { B: barbitúricos } \\
\text { C: carbamazepina y ciclosporina } \\
\text { D: digitálicos y dapsona }\end{array}$ & $\begin{array}{l}\text { Para HD: xenobiótico de bajo } \\
\text { peso molecular }(<500 \mathrm{Da}) \text {, } \\
\text { hidrosoluble y con baja unión a } \\
\text { proteínas }(<30 \%) \\
\text { Para HF: peso molecular }<10,000 \\
\text { Da, baja unión a proteínas } \\
(<30 \%) \\
\text { Para HP: absorbible por CA }\end{array}$ \\
\hline Contraindicaciones & $\begin{array}{l}\text { Insuficiencia renal, alcalosis o } \\
\text { hipopotasemia (relativas) }\end{array}$ & $\begin{array}{l}\text { Xenobiótico no absorbible por CA, } \\
\text { cáusticos y riesgo de sangrado o } \\
\text { perforación }\end{array}$ & Inestabilidad hemodinámica \\
\hline Técnica & $\begin{array}{l}\text { Bicarbonato de sodio: bolo } \\
\text { inicial de } 1 \text { a } 2 \mathrm{mEq} / \mathrm{kg} \text { de peso, } \\
\text { seguido infusión continua de } 0.3 \\
\text { a } 0.5 \mathrm{mEq} / \mathrm{kg} / \mathrm{h} \text { diluidos en } \\
\text { solución glucosada al } 5 \% \text { en } \\
\text { una relación } 1: 5 . \text { El objetivo es } \\
\text { mantener un pH sérico entre } 7.5 \\
\text { y } 7.55 \text { para un pH urinario de } \\
\text { entre } 7.5 \text { y } 8\end{array}$ & $\begin{array}{l}\text { 1. Verificar reflejos de la vía aérea o } \\
\text { intubar } \\
\text { 2. Elegir la vía de administración (oral o } \\
\text { por sonda) } \\
\text { 3. Dosis inicial de } 1 \mathrm{~g} / \mathrm{kg} \text { de peso, dosis } \\
\text { de mantenimiento de } 0.5 \mathrm{~g} / \mathrm{kg} \text { cada } 4 \\
\text { a } 6 \text { horas } \\
\text { 4. La dosis inicial se da con catártico y } \\
\text { las subsecuentes se valorará } \\
\text { dependiendo la función intestinal del } \\
\text { paciente } \\
\text { 5. Siempre se verifica el abdomen } \\
\text { antes de la siguiente dosis } \\
\text { 6. La técnica concluye al lograr el } \\
\text { resultado esperado o si las } \\
\text { condiciones abdominales lo impiden } \\
\text { (aumento de presión intraabdominal, } \\
\text { íleo, oclusión o sangrado) }\end{array}$ & $\begin{array}{l}\text { 1. Colocación de catéter central } \\
\text { 2. Solicitar abordaje por parte de } \\
\text { servicio de nefrología- } \\
\text { hemodiálisis } \\
\text { 3. La anticoagulación, así como } \\
\text { los flujos, membranas y } \\
\text { duración del tratamiento } \\
\text { dependen de la técnica } \\
\text { empleada, el calibre del } \\
\text { catéter central, el xenobiótico } \\
\text { que se pretende aclarar y el } \\
\text { estado clínico del paciente }\end{array}$ \\
\hline Complicaciones & $\begin{array}{l}\text { Alcalosis, hipopotasemia, } \\
\text { hipernatremia }\end{array}$ & $\begin{array}{l}\text { Náusea, vómitos, cólico, malestar } \\
\text { gastrointestinal, neumonitis por } \\
\text { aspiración, formación de bezoares y } \\
\text { oclusión intestinal }\end{array}$ & $\begin{array}{l}\text { Hemorragias, bacteriemia, } \\
\text { alteraciones electrolíticas, } \\
\text { hemólisis, hipotensión y } \\
\text { aclaramiento de medicamentos }\end{array}$ \\
\hline
\end{tabular}

CA: carbón activado; HD: hemodiálisis; HF: hemofiltración; HP: hemoperfusión.

Adaptada de Goldfarb, et al., 201920; Proudfoot, et al., 2004²1; Bradberry, et al., 199522; Chyka, et al., 199523; American Academy of Clinical Toxicology, European Association of Poisons Centers and Clinical Toxicologists, 199924; Holubek, et al., 200825; Bismuth, 199026; Borkan, 200227; Goodman, et al., 200628.

xenobiótico es susceptible de ser removido mediante las técnicas disponibles, lo cual depende de las características propias de cada xenobiótico (tamaño de la molécula, volumen de distribución, constante de disociación, unión a proteínas, aclaramiento renal, etc.). Los métodos que se utilizan para incrementar la eliminación de los xenobióticos se pueden clasificar en métodos intrínsecos o corpóreos y métodos extracorpóreos y se describen en la tabla $3^{20-28}$.

La diálisis peritoneal tiene una capacidad de aclaramiento muy limitada para la mayoría de los xenobióticos, por lo que no se recomienda su uso en toxicología a no ser que no se disponga de otra técnica ${ }^{29}$. Por su parte, la plasmaféresis y la exanguinotransfusión son métodos empleados para la eliminación de moléculas de gran tamaño (>150,000 Da), como amatoxinas, tiroxina, vincristina y complejos inmunes (p. ej., digoxina) o de moléculas adheridas a elementos sanguíneos como el gas arsina, además de aquellos casos donde no son aplicables otras técnicas de eliminación o estas son riesgosas, como sucede en el recién nacido o 
Tabla 4. Antídotos

\begin{tabular}{|c|c|c|c|}
\hline Antídoto & Indicaciones & Posología & Efectos adversos \\
\hline Acetilcisteína & $\begin{array}{l}\text { Intoxicación por: paracetamol, } \\
\text { tetracloruro de carbono y } \\
\text { cloroformo }\end{array}$ & $\begin{array}{l}\text { Oral. Inicial: } 140 \mathrm{mg} / \mathrm{kg} \mathrm{y} \\
\text { mantenimiento: } 70 \mathrm{mg} / \mathrm{kg} \mathrm{c} / 4 \mathrm{~h} \\
\text { (17 dosis). Total: } 72 \mathrm{~h} \text { (18 dosis). } \\
\text { Intravenoso. Inicial: } 150 \mathrm{mg} / \mathrm{kg} \text {, } \\
\text { para } 30-60 \text { min, segunda dosis: } \\
50 \mathrm{mg} / \mathrm{kg} \text { para } 4 \mathrm{~h} \text {, tercera dosis: } \\
100 \mathrm{mg} / \mathrm{kg} \text { para } 16 \mathrm{~h}\end{array}$ & $\begin{array}{l}\text { Náusea, vómitos, diarrea y dolor } \\
\text { abdominal (oral), reacciones alérgicas, } \\
\text { cefalea, fiebre y tinnitus }\end{array}$ \\
\hline Ácido folínico & $\begin{array}{l}\text { Intoxicación por: metotrexato y } \\
\text { metanol }\end{array}$ & $\begin{array}{l}10-25 \mathrm{mg} / \mathrm{m}^{2} \text { cada } 4-6 \mathrm{~h} \text { por un } \\
\text { mínimo de } 72 \mathrm{~h}\end{array}$ & Reacciones de hipersensibilidad \\
\hline Atropina & $\begin{array}{l}\text { Intoxicación por: } \\
\text { colinérgicos (organofosforados, } \\
\text { carbamatos y agentes nerviosos) }\end{array}$ & $\begin{array}{l}\text { Adultos: } 1-3 \mathrm{mg} / \mathrm{dosis} \\
\text { Niños: } 0.02-0.05 \mathrm{mg} / \mathrm{kg} / \text { dosis, cada } \\
3-5 \text { minutos }\end{array}$ & $\begin{array}{l}\text { Midriasis, taquicardia, arritmias, visión } \\
\text { borrosa, sequedad de mucosas, íleo, } \\
\text { retención urinaria }\end{array}$ \\
\hline Azul de metileno & MetHb & $\begin{array}{l}1-2 \mathrm{mg} / \mathrm{kg} \mathrm{IV} \text {, se puede repetir la } \\
\text { dosis a los } 30-60 \text { minutos }\end{array}$ & $\begin{array}{l}\text { Náusea, vómitos, disnea, hipertensión, } \\
\text { confusión, si se utilizan a dosis }>7 \mathrm{mg} / \mathrm{kg} \text {, } \\
\text { puede producir hemólisis y MetHb }\end{array}$ \\
\hline Calcio & $\begin{array}{l}\text { Intoxicación por: etilenglicol, } \\
\text { fluoruros, oxalatos, } \\
\text { calcioantagonistas y } \\
\text { betabloqueadores }\end{array}$ & $\begin{array}{l}10-20 \mathrm{mg} / \mathrm{kg} \text { IV en niños y } 1 \mathrm{~g} \\
(50 \mathrm{mEq} \text { ) en adultos. Administrar } \\
\text { en } 10-15 \text { min y cada } 5 \text { min hasta } \\
\text { obtener respuesta }\end{array}$ & $\begin{array}{l}\text { Hipercalcemia, náusea, vómitos, } \\
\text { hipotensión, depresión miocárdica, } \\
\text { trastornos del ritmo cardiaco. En } \\
\text { extravasación produce dolor, irritación } \\
\text { química y necrosis local }\end{array}$ \\
\hline I-carnitina & Intoxicación por: ácido valproico & $\begin{array}{l}50-500 \text { mg/kg/día IV en } 4 \text { dosis. } \\
\text { Niños: dosis máxima } 6 \mathrm{~g} / \text { día }\end{array}$ & $\begin{array}{l}\text { Náusea, vómitos, diarrea y a dosis altas } \\
\text { un olor corporal a pescado }\end{array}$ \\
\hline Ciproheptadina & Síndrome serotoninérgico & $\begin{array}{l}\text { Dosis inicial: } 12 \text { mg por vía oral, } \\
\text { seguida de } 2 \text { mg cada } 2 \text { horas } 0 \\
\text { de } 4 \text { a } 8 \text { mg cada } 6 \text { horas hasta } \\
\text { obtener el control de los síntomas }\end{array}$ & $\begin{array}{l}\text { Sedación, mareo, incoordinación, } \\
\text { insomnio, temblor, euforia, ansiedad y } \\
\text { crisis convulsivas }\end{array}$ \\
\hline Dantroleno & $\begin{array}{l}\text { Hipertermia maligna y síndrome } \\
\text { neuroléptico maligno }\end{array}$ & $\begin{array}{l}\text { Inicial } 2.5 \mathrm{mg} / \mathrm{kg} \text { IV en bolo rápido, } \\
\text { mantenimiento: } 1 \mathrm{mg} / \mathrm{kg} \text { cada } 4 \text { a } \\
6 \text { horas o infusión continua a } \\
0.25 \mathrm{mg} / \mathrm{kg} / \mathrm{h}\end{array}$ & $\begin{array}{l}\text { Sedación, debilidad persistente y lesión } \\
\text { hepática aguda (rara) }\end{array}$ \\
\hline $\begin{array}{l}\text { Emulsión de } \\
\text { lípidos }\end{array}$ & $\begin{array}{l}\text { Intoxicación por: anestésicos } \\
\text { locales, calcioantagonistas, } \\
\text { betabloqueadores, } \\
\text { antidepresivos } \\
\text { cíclicos, clorpromazina y } \\
\text { lamotrigina }\end{array}$ & $\begin{array}{l}\text { Bolo de } 1-1.5 \mathrm{ml} / \mathrm{kg} \text { en } 1 \mathrm{a} \\
3 \text { minutos, mantenimiento con } \\
\text { infusión de } 0.25-0.50 \mathrm{ml} / \mathrm{kg} / \mathrm{min} \text {, } \\
\text { para una hora }\end{array}$ & $\begin{array}{l}\text { Reacciones de hipersensibilidad, } \\
\text { trombocitopenia, aumento de actividad } \\
\text { antineutrofílica y plaquetaria, } \\
\text { infecciones, hipertermia, pancreatitis, } \\
\text { embolia grasa, elevación de } \\
\text { transaminasas y triglicéridos }\end{array}$ \\
\hline Etanol & $\begin{array}{l}\text { Intoxicación por: } \\
\text { alcoholes (metanol y etilenglicol) }\end{array}$ & $\begin{array}{l}\text { En concentración } 10-20 \% \text { : } \\
\text { Inicial: } 800 \mathrm{mg} / \mathrm{kg} \text { V0 en } \\
20 \text { minutos } \\
\text { Mantenimiento: } 80-150 \mathrm{mg} / \mathrm{kg} / \mathrm{h} \\
\text { para mantener la concentración } \\
\text { sérica entre } 100-150 \mathrm{mg} / \mathrm{dl}\end{array}$ & $\begin{array}{l}\text { Depresión del SNC, hipoglucemia, } \\
\text { gastritis, pancreatitis y toxicidad } \\
\text { hepática }\end{array}$ \\
\hline Fisostigmina & Intoxicación por: anticolinérgicos & $\begin{array}{l}1-2 \mathrm{mg} \text { en adultos y } 0.02 \mathrm{mg} / \mathrm{kg} \text { en } \\
\text { niños (máximo } 0.5 \mathrm{mg} \text { ) en bolo } \\
\text { lento }\end{array}$ & $\begin{array}{l}\text { Efectos muscarínicos: vómitos, } \\
\text { sudoración, hipersalivación, } \\
\text { diarrea, miosis, lagrimeo e } \\
\text { incontinencia urinaria y fecal }\end{array}$ \\
\hline Flumazenilo & $\begin{array}{l}\text { Contrarrestar efectos de } \\
\text { benzodiazepinas siempre que no } \\
\text { exista: consumo crónico de } \\
\text { benzodiazepinas u otros } \\
\text { sedantes, epilepsia, consumo de } \\
\text { proconvulsivantes, trauma de } \\
\text { cráneo, alteraciones en EKG, } \\
\text { hipoxia, hipotensión o } \\
\text { desconocimiento de los } \\
\text { antecedentes del paciente }\end{array}$ & $0.2 \mathrm{mg}$ IV o $0.01 \mathrm{mg} / \mathrm{kg}$ en niños & $\begin{array}{l}\text { Náusea, vómitos, ansiedad, agitación, } \\
\text { síndrome de abstinencia, crisis } \\
\text { convulsivas y arritmias }\end{array}$ \\
\hline
\end{tabular}


Tabla 4. Antídotos (continuación)

\begin{tabular}{|c|c|c|c|}
\hline Antídoto & Indicaciones & Posología & Efectos adversos \\
\hline Fomepizol & $\begin{array}{l}\text { Intoxicación por: } \\
\text { alcoholes (metanol y etilenglicol) }\end{array}$ & $\begin{array}{l}\text { Inicial: } 15 \mathrm{mg} / \mathrm{kg} \\
\text { Mantenimiento: } 10 \mathrm{mg} / \mathrm{kg} \text { cada } \\
12 \mathrm{~h} \\
\text { Luego de } 48 \mathrm{~h} \text { la dosis de reajusta } \\
\text { a } 15 \mathrm{mg} / \mathrm{kg} \text { de requerir continuar } \\
\text { el tratamiento }\end{array}$ & $\begin{array}{l}\text { Cefalea, náusea, vértigo, ansiedad, } \\
\text { flebitis, rash, eosinofilia, elevación } \\
\text { transitoria de las transaminasas }\end{array}$ \\
\hline Glucagón & $\begin{array}{l}\text { Intoxicación por: } \\
\text { betabloqueadores, } \\
\text { calcioantagonistas }\end{array}$ & $\begin{array}{l}\text { Inicial: adultos } 3-5 \text { mg (máx. } 10 \mathrm{~g} \text { ) } \\
\text { y niños } 50 \mu \mathrm{g} / \mathrm{kg} 0 \text { infusión: } \\
50-100 \mu \mathrm{g} / \mathrm{kg} / \mathrm{h}\end{array}$ & $\begin{array}{l}\text { Náusea, vómitos, hiperglucemia, } \\
\text { hipoglucemia e hipocalcemia }\end{array}$ \\
\hline Insulina & $\begin{array}{l}\text { Intoxicación por: } \\
\text { calcioantagonistas y } \\
\text { betabloqueadores }\end{array}$ & $\begin{array}{l}1 \mathrm{UI} / \mathrm{kg} \text { en bolo y una infusión de } \\
0.5 \mathrm{U} / \mathrm{kg} / \mathrm{h} \text {, ajustando } \\
\text { dosis-respuesta. Combinar con } \\
\text { una infusión de glucosa a } 1 \mathrm{~g} / \mathrm{kg} / \mathrm{h}\end{array}$ & $\begin{array}{l}\text { Trastornos del metabolismo de la } \\
\text { glucemia (hipoglucemia e } \\
\text { hiperglucemia) }\end{array}$ \\
\hline Naloxona & $\begin{array}{l}\text { Intoxicación por opioides } \\
\text { siempre que no exista hipoxia }\end{array}$ & $\begin{array}{l}\text { Inicial: } 0.04 \text { mg IV, incrementando } \\
\text { a } 0.4 \mathrm{mg}, 2 \mathrm{mg} \text { y } 10 \mathrm{mg} \text { cada } 2 \mathrm{a} \\
5 \text { minutos si no existe respuesta } \\
\text { Mantenimiento: } 2 / 3 \text { de la dosis } \\
\text { necesaria para revertir la } \\
\text { depresión respiratoria cada } \\
\text { hora (en bolo o infusión) }\end{array}$ & $\begin{array}{l}\text { Síndrome de } \\
\text { abstinencia en usuarios crónicos, } \\
\text { edema pulmonar no cardiogénico, } \\
\text { hipertensión, hipotensión, arritmias y } \\
\text { convulsiones }\end{array}$ \\
\hline Obidoxima & $\begin{array}{l}\text { Intoxicación por: insecticidas } \\
\text { oganofosforados y agentes } \\
\text { nerviosos (sarín, tabún, somán, } \\
\text { VX) }\end{array}$ & $\begin{array}{l}\text { Bolo inicial de } 250-500 \mathrm{mg} \text { en el } \\
\text { adulto y de } 4-8 \mathrm{mg} / \mathrm{kg} \text { en niños en } \\
\text { bolo lento } \\
\text { Mantenimiento: infusión continua } \\
\text { o cada } 4-6 \mathrm{~h} \text { una dosis de } \\
750 \mathrm{mg} / 24 \mathrm{~h} \text { en el adulto y } \\
10 \mathrm{mg} / \mathrm{kg} / \text { día en el niño }\end{array}$ & $\begin{array}{l}\text { Signos anticolinérgicos, náusea, } \\
\text { cefalea, taquicardia, alteraciones } \\
\text { visuales, debilidad muscular y asistolia }\end{array}$ \\
\hline $\begin{array}{l}\text { Oxígeno } \\
\text { hiperbárico }\end{array}$ & $\begin{array}{l}\text { Intoxicaciones por: monóxido de } \\
\text { carbono, cianuro y loxoscelismo } \\
\text { dermonecrótico (terapia } \\
\text { adyuvante) }\end{array}$ & $\begin{array}{l}\text { 2-3 atmósferas a tolerancia del } \\
\text { paciente. Suele requerirse un } \\
\text { mínimo de } 3 \text { a } 5 \text { sesiones }\end{array}$ & $\begin{array}{l}\text { Barotrauma, principalmente de oído } \\
\text { medio, neumotórax, embolia gaseosa, } \\
\text { enfermedad por descompresión y crisis } \\
\text { convulsivas }\end{array}$ \\
\hline d-penicilamina & $\begin{array}{l}\text { Intoxicación por: metales (plomo, } \\
\text { zinc, cobre, oro, arsénico y } \\
\text { mercurio) }\end{array}$ & 20-100 mg/kg/día V0 & $\begin{array}{l}\text { Reacciones de } \\
\text { hipersensibilidad (urticaria, } \\
\text { angioedema), leucocitopenia, } \\
\text { eosinofilia, trombocitopenia, náuseas y } \\
\text { vómitos }\end{array}$ \\
\hline $\begin{array}{l}\text { Piridoxina } 0 \\
\text { vitamina B6 }\end{array}$ & $\begin{array}{l}\text { Intoxicación por: isoniazida, } \\
\text { setas (gyromitra, muscimol) y } \\
\text { etilenglicol }\end{array}$ & $\begin{array}{l}\text { La dosis de piridoxina es la misma } \\
\text { que la dosis ingerida de } \\
\text { isoniazida ( } 1 \mathrm{~g} \text { de piridoxina=1 } \mathrm{g} \\
\text { de isoniazida). Dosis empírica: en } \\
\text { adultos } 5 \mathrm{~g} \text { de piridoxina IV en } \\
3-5 \text { minutos, niños } 70 \mathrm{mg} / \mathrm{kg} \text { hasta } \\
\text { revertir el efecto }\end{array}$ & $\begin{array}{l}\text { Con dosis altas puede presentar } \\
\text { convulsiones, parálisis, taquipnea y } \\
\text { neuropatía sensitiva }\end{array}$ \\
\hline $\begin{array}{l}\text { Vitamina K1 o } \\
\text { fitomenadiona }\end{array}$ & $\begin{array}{l}\text { Intoxicación por: anticoagulantes } \\
\text { warfarínicos (medicamentos y } \\
\text { rodenticidas) }\end{array}$ & $\begin{array}{l}\text { IV/IM: niños de } 1 \text { a } 5 \mathrm{mg} / \text { día y en } \\
\text { adultos } 10 \mathrm{mg} / \text { día por } 3 \text { a } 5 \text { días }\end{array}$ & $\begin{array}{l}\text { Reacciones de hipersensibilidad, } \\
\text { hipotensión, disnea, cianosis, mareo, } \\
\text { hiperbilirrubinemia, anemia hemolítica, } \\
\text { dolor local y edema }\end{array}$ \\
\hline
\end{tabular}

EKG: electrocardiograma; IV: intravenoso; V0: vía oral; IM: intramuscular; MetHb: metahemoglobinemia; SNC: sistema nervioso central. Adaptada de Smith, et al., 201932; Betten, et al., 200633; Dart, et al., 200934; Nogué, et al., 201535; Ferrer, et al., 199736; Pettit, et al., $1999^{37}$.

lactante, que poseen un menor volumen circulante. En ambos casos se extrae sangre total del paciente, por lo que es indispensable que el xenobiótico tenga un modelo de distribución monocompartimental y un bajo volumen de distribución. Desafortunadamente su poca disponibilidad, la complejidad de su realización y sus limitadas aplicaciones las han hecho caer en el desuso ${ }^{20}$. 


\section{Antídoto}

Un antídoto puede ser definido como una sustancia que es administrada específicamente para contrarrestar los efectos nocivos de un tóxico o una sustancia potencialmente tóxica, mediante una acción química relativamente específica. Contrario a lo que pudiera pensarse, el empleo de antídotos está reducido a un pequeño número de sustancias tóxicas y en la mayoría de los casos se reserva para aquellos pacientes en los que no están disponibles otras formas de tratamiento o estas fracasaron. Las excepciones son la intoxicación por paracetamol y los envenenamientos para los que se dispone de faboterápico específico (ofidios y arácnidos), entre los de uso más frecuente. En otros casos, el antídoto puede desencadenar síndromes de abstinencia (p. ej., flumazenilo y naloxona) o inducir efectos tóxicos propios (p. ej., fisostigmina), por lo que la decisión sobre la utilización de un antídoto depende del estado clínico del paciente, su especificidad y la evaluación juiciosa del riesgo-beneficio en cada caso ${ }^{30,31}$. Para ello debe considerarse siempre la premisa de «tratar al paciente y no al tóxico». Adicionalmente, es importante tener en cuenta las características farmacocinéticas del tóxico y del antídoto, ya que, en ocasiones, la vida media de algunos antídotos es más corta, lo que puede provocar la recurrencia de los síntomas de intoxicación ${ }^{32}$. En la tabla 4 se presentan los principales antídotos y sus aplicaciones para el tratamiento de un paciente potencialmente intoxicado ${ }^{32-37}$.

\section{Agradecimientos}

Agradecemos a la Dra. Janett Santos Sánchez, por haber sentado las bases para la formación física y académica de este centro toxicológico para quienes hoy laboramos en él.

\section{Financiamiento}

El presente artículo no ha recibido ninguna beca específica de agencias de los sectores públicos, comercial o sin ánimos de lucro.

\section{Conflicto de intereses}

Los autores declaran no tener conflicto de intereses.

\section{Responsabilidades éticas}

Protección de personas y animales. Los autores declaran que para esta investigación no se han realizado experimentos en seres humanos ni en animales.

Confidencialidad de los datos. Los autores declaran que en este artículo no aparecen datos de pacientes.

Derecho a la privacidad y consentimiento informado. Los autores declaran que en este artículo no aparecen datos de pacientes.

\section{Bibliografía}

1. Kuling K. Initial management of ingestions of toxic substances. N Engl J Med. 1992;326:1677-81.

2. Erickson TB, Goldfrank LR, Kuling K. How to treat the poisoned patient? Patient care. 1997;90:90-113.

3. Schwarz ES. Therapeutic approach to the critically poisoned patient. En: Brent J. Critical care toxicology. Diagnosis and management of the critically poisoned patient. $2^{\text {nd }}$ ed. EE.UU.: Springer; 2017. pp. 43-78.

4. Hoegberg LCG. Techniques used to prevent gastrointestinal absorption. En: Nelson LS, Howland RS, Howland MA, Lewin NA, Goldfrank LR, et al. Goldfrank's toxicologic emergencies. $11^{\text {th }}$ ed. EE.UU.: Mc Graw-HiII; 2019. pp. 48-70.

5. Chyka PA, Seger D, Krenzelok EP, Vale JA; American Academy of Clinical Toxicology; European Association of Poisons Centres and Clinical Toxicologists. Position paper: single-dose activated charcoal. Clin Toxicol. 2005;43(2):61-87.

6. Albertson TE, Owen KP, Sutter ME, Chan AL. Gastrointestinal decontamination in the acutely poisoned patient. Int J Em Med. 2011;4:65.

7. Merigian KS, Woodard M, Hedges JR, Roberts JR, Stuebing R, Rashkin MC. Prospective evaluation of gastric emptying in the self-poisoned patient. Am J Emerg Med. 1990;8(6):479-83.

8. Harchelroad F, Cottington E, Krenzelok EP. Gastrointestinal transit times of a charcoal/sorbitol slurry in overdose patients. Clin Toxicol. 1989;27(12):90-9.

9. Green R, Sitar DS, Tenenbein M. Effect of anticholinergic drugs on the efficacy of activated charcoal. J Toxicol Clin Toxicol. 2004;42(3):267-72.

10. Benson BE, Hoppu K, Troutman WG, Bedry R, Erdman A, Höjer J, et al; American Academy of Clinical Toxicology; European Association of Poisons Centres and Clinical Toxicologists. Position paper update: gastric lavage for gastrointestinal decontamination. Clin Toxicol. 2013;51(3):140-6.

11. Phillips S, Gomez H, Brent J. Pediatric gastrointestinal decontamination in acute toxin ingestion. J Clin Pharmacol. 1993;33(6):497-507.

12. Perrone J, Hoffman RS, Goldfrank LR. Special considerations in gastrointestinal decontamination. Emerg Med Clin North Am. 1994;12(2):285-99.

13. Buckley NA, Eddleston M. The revised position papers on gastric decontamination. Clin Toxicol. 2005;43(2):129-30.

14. Thanacoody R, Caravati EM, Troutman B, Höjer J, Benson B, Hoppu K, et al. Position paper update: Whole bowel irrigation for gastrointestinal decontamination of overdose patients. Clin Toxicol. 2015;53(1):5-12.

15. Pérez TJ, Pérez HJ, Bautista AM. Extended release potassium salts overdose and endoscopic removal of a pharmacobezoar: a case report. Tox Rep. 2014;1:209-13.

16. United States Environmental Protection Agency (EPA). Personal protective equipment [Internet]. United States Environmental Protection Agency [acceso: 5 de febrero de 2020]. Disponible en: https://www.epa.gov/ emergency-response/personal-protective-equipment

17. Lucyk S. Decontamination principles: prevention of dermal, ophthalmic and inhalational absorption. En: Nelson LS, Howland RS, Howland MA, Lewin NA, Goldfrank LR, et al. Goldfrank's toxicologic emergencies. $11^{\text {th }}$ ed. EE.UU.: Mc Graw-Hill; 2019. pp. 71-5.

18. Casavant MJ. Assessment of clinical policy for the initial approach to patients presenting with acute toxic ingestion or dermal or inhalation exposure. Ann Emerg Med. 2000;35(2):196-7.

19. Flach AJ. Systemic toxicity associated with topical ophthalmic medications. J Fla Med Assoc. 1994;81(4):256-60.

20. Goldfarb DS, Ghannoum M. Principles and techniques applied to enhance elimination. En: Nelson LS, Howland RS, Howland MA, Lewin NA, Goldfrank LR, et al. Goldfrank's toxicologic emergencies. $11^{\text {th }}$ ed. EE. UU.: Mc Graw-Hill; 2019. pp. 90-100.

21. Proudfoot AT, Krenzelok EP, Vale JA. Position paper on urine alkalization. J Toxicol Clin Toxicol. 2004;42(1):1-26.

22. Bradberry SM, Vale JA. Multiple-dose activated charcoal: A review of relevant clinical studies. Clin Toxicol. 1995;33(5):407-16.

23. Chyka PA, Holley JE, Mandrell TD, Sugathan P. Correlation of drug pharmacokinetics and effectiveness of multiple-dose activated charcoal therapy. Ann Emerg Med. 1995;25(3):356-62. 
24. American Academy of Clinical Toxicology, European Association of Poisons Centers and Clinical Toxicologists. Position statement and practice guidelines on the use of multi-dose activated charcoal in the treatment of acute poisoning. Clin Toxicol. 1999;37(6):731-51.

25. Holubek WJ, Hoffman RS, Goldfarb DS, Nelson LS. Use of hemodialysis and hemoperfusión in poisoned patients. Kidney Int 2008;74(10):1327-34

26. Bismuth $\mathrm{C}$. Biological valuation of extra-corporeal techniques in acute poisoning. Acta Clin Belg Suppl. 1990;13:20-8.

27. Borkan SC. Extracorporeal therapies for acute intoxications. Crit Care Clin. 2002;18(2):393-420.

28. Goodman JW, Goldfarb DS. The role of continuous renal replacement therapy in the treatment of poisoning. Semin Dial. 2006;19(5):402-7.

29. Keyvan-Larijarni H, Tannenberg AM. Methanol intoxication: comparison of peritoneal dialysis and hemodialysis treatment. Arch Intern Med. 1974;134(2):293-6.

30. Barnett R, Grace M, Boothe P, Latozek K, Neal C, Legatt D, et al. Flumazenil in drug overdose: randomized, placebo-controlled study to assess cost effectiveness. Crit Care Med. 1999;27:78-81.
31. Hoffman JR, Schiger DL. The empiric use of naloxone in patients with altered mental status: a reappraisal. Ann Emerg Med. 1991;20:246-52.

32. Smith SW, Goldfrank LR, Howland MA. Principles on antidote stocking En: Nelson LS, Howland RS, Howland MA, Lewin NA, Goldfrank LR, et al. Goldfrank's toxicologic emergencies. $11^{\text {th }}$ ed. EE.UU.: Mc Graw-HiII; 2019. pp. 42-7.

33. Betten D, Vohra RB, Cook MD, Matteucci MJ, Clark RF. Antidote use in the critically ill poisoned patient. J Intensive Care Med. 2006;21(5):255-77.

34. Dart RC, Borron SW, Caravati EM, Cobaugh DJ, Curry SC, Falk JL, et al. Antidote Summit Authorship Group. Expert consensus guidelines for stocking of antidotes in hospitals that provide emergency care. Ann Emerg Med. 2009;54(3):386-94.

35. Nogué S, Miró O, Aguilar R. Emergency antidotes and services. An Pediatr (Barc). 2015;82(5):260-1

36. Ferrer A, Civeira E, Lopez P, Loren B. The use of antidotes in the management of central nervous system depression. Arch Toxicol Suppl. 1997;19:289-98.

37. Pettit HE, McKinney PE, Achusim LE, Lindsay DC. Toxicology cart for stocking sufficient supplies of poisoning antidotes. Am J Health Syst Pharm. 1999;56(24):2537-9. 